\title{
Performance Analysis of MIMO-OFDM Systems on Coexistence Environment in FDD-LTE Networks
}

\author{
S. Malisuwan, J. Sivaraks, N. Tiamnara, and Y. Thamachareon
}

\begin{abstract}
The explosive growth of Multiple Input Multiple Output (MIMO) systems has recently drawn wide interests due to their capability of high data rate and wide variety of applications. Recent advances in Long Term Evolution (LTE) communication systems have contributed to the design of multi-user scenarios with MIMO communication. This paper addresses the effect of coexistence of FDD-LTE networks in 2.6GHz frequency band employing MIMO-OFDM antenna systems in Rayleigh channel. The performance results of $2 \times 2$ MIMO-OFDM systems on the coexistence of FDD-LTE Networks is evaluated by using simulations on MATLAB.
\end{abstract}

Index Terms-MIMO-OFDM, performance, coexistence, FDD-LTE.

\section{INTRODUCTION}

Transmission of data in wireless communication systems is extensively discussed due to exponential demand and rise of multimedia wireless communication applications. The difficulty in wireless communication lies in phenomena called multipath induced fading also called random fluctuation in the channel gain, which occurs as a result of multipath scattering. Earlier, multipath scattering was the biggest drawback in wireless communication however, due to increase in use of multiple antennas in the transmitter and receiver called MIMO, rich scattering environment can be exploited to create multiplicity of parallel links to increase the data rate without the expense of extra bandwidth [1].

"Main issue encountered when deploying mobile communication networks is coexistence and/or collocation problem, that is, inter-cell interference between mobile networks themselves" [2]. This has been addressed quite extensively in the literature [3]-[5]. Although Long Term Evolution (LTE) is currently being deployed extensively worldwide, many radio aspects impacting LTE coexistence conditions have been studied in very few research [2], [6]. As MIMO is one aspect that impacts LTE deployment but has rarely been studied, this research seeks to investigate and model Performance Analysis of MIMO-OFDM Systems on Coexistence Environment in FDD-LTE Networks [7]. There are two types of inter-cell interference, interference on the same frequency is Co-Channel Interference $(\mathrm{CCI})$ and on adjacent frequencies is called Adjacent Channel Interference (ACI) [2]. In Co-Channel Interference, receiver desensitization may occur either together or separately as:

Manuscript received March 5, 2014; revised May 7, 2014. This work was financially supported by National Broadcasting and Telecommunications Commission, Bangkok, Thailand.

The authors are with National Broadcasting and Telecommunications Commission Bangkok, Thailand (e-mail: settapong.m@nbtc.go.th, jesada.s@nbtc.go.th,noppadol.m@nbtc.go.th, nattakit.s@nbtc.go.th).
Out-of-Band Emissions (OOBE) and receiver overload (such as blocking and inter-modulation).

OOBE occurs when transmission of a signal goes beyond the intended channel causing disturbance to victim receiver. The strongest impact is on contiguous channels which is Adjacent Channel Interference (ACI) [2].

Receiver overload is when blocking occurs within the receiver, or co-channel interference occurs due to large signals within the receiver front-end passband. The signals can be near or in the receiver where it cause disturbance and they can also be anywhere within the front end passband of the receiver that is disturbed [8].

OFDM stands for orthogonal frequency division multiplexing, it is the specific type of multicarrier modulation scheme. OFDM is division of available spectrum into several sub channels hence, each sub channel an act as narrow band experiencing flat fading [9]. OFDM is based on DFT to generate orthogonal carriers, hence the name OFDM. In this way the frequency response of sub channels are overlapped, effectively results in high spectral efficiency of transmission system.

MIMO-OFDM offers simplicity in decoding, while it has many advantages that makes it a preferable choice, it also poses some challenges. MIMO-OFDM symbol is typically long while compared to a single carrier. So in practical system even there is small channel variation over OFDM symbol, the orthogonality of subcarriers is affected which may result in inter-cell interference which will result in degradation of system performance. This paper presents the performance evaluation of $2 \times 2$ MIMO-OFDM systems on coexistence environment in LTE networks. The paper focuses mainly on the interference in FDD-LTE Downlink $(2620 \mathrm{MHz}-2625 \mathrm{MHz})$ as shown in Fig.1.

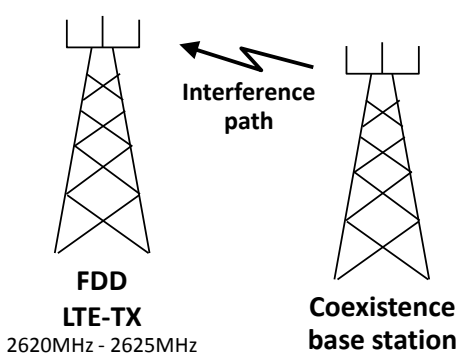

Fig. 1. LTE inter-cell interference within $2.6 \mathrm{GHz}$ frequency band.

\section{INTER-CELL INTERFERENCE}

The following model is used to assess the inter-cell power between LTE cells:

$$
\begin{gathered}
I=P_{T x}+G_{T x}+G_{R x}+10 \log (N)-A C I R-32.4- \\
20 \log (f)-20 \log (d)-A_{h}
\end{gathered}
$$


As presented in [2] " $I$ is the interference $(\mathrm{dBm})$ that is transmitted from the cell causing the interference to the cell that disturbed, $P$ is transmission power $(\mathrm{dBm})$ of interfering cell, $G_{T x}$ and $G_{T x}$ are interferer transmitter and victim receiver antenna gains $(\mathrm{dBi})$. While $N$ is number of transmitting antenna elements employed by interfering transmitter $f$ is radio frequency $(\mathrm{MHz})$ of interfering transmitter, $d$ is the distance $(\mathrm{km})$ between interfering and victim cells, $A C I R$ and $A_{h}$ are ACI power ratio and deployment environment clutter loss."

Adjacent Channel Interference Ratio (ACIR) is the total amount of leak experienced between two transmissions on adjacent channels and derived from Adjacent Channel Leakage Ratio (ACLR) and Adjacent Channel Selectivity $(\mathrm{ACS})$ as seen in equation below [10]:

$$
A C I R=\frac{1}{\frac{1}{A C L R}+\frac{1}{A C S}}
$$

The $A_{h}$ is a total loss owing to nearby clutter and is indicated as:

$$
A_{h}=10.25 e^{-d_{k}}\left[1-\tan h\left[6\left(\frac{h}{h_{a}}-0.625\right)\right]\right]-0.33
$$

where $d_{k}(\mathrm{~km})$ distance between nearby clutters and the antenna that cause interference and $h_{a}$ and $h$ are the nominal clutter height and height of antenna experiencing interference as indicated in Table I [11].

\section{TABLE I: CluTter HEIGHTS AND DistANCES}

\begin{tabular}{ccc}
\hline \hline Clutter Category & Clutter Height $\mathrm{h}_{\mathrm{a}}(\mathrm{m})$ & Nominal Distance $\mathrm{d}_{\mathrm{k}}(\mathrm{km})$ \\
\hline Rural & 4 & 0.1 \\
Suburban & 9 & 0.025 \\
Urban & 20 & 0.02 \\
Dense urban & 25 & 0.02 \\
\hline \hline
\end{tabular}

System degradation is the performance degradation occurred as a result of receiver sensitivity and hence assessed as $(\mathrm{S})$ and calculated as the noise rise due to the received interference [11]:

$$
S=10 \log \left(\frac{10^{\frac{N}{10}}+10^{\frac{I}{10}}}{10^{\frac{N}{10}}}\right)=10 \log \left(1+10^{\frac{I-N}{10}}\right)
$$

where $S$ is degradation of receiver sensitivity $(\mathrm{dB}), I$ is the received interference $(\mathrm{dBm})$ as indicated in Eqn.(1) and $N$ is victim receiver noise floor $(\mathrm{dBm})$, and is given by

$$
N=N_{\text {thermal }}+N_{\text {figure }}+10 \log (B W)
$$

where $N_{\text {thermal }}$ and $N_{\text {figure }}$ are "victim receiver thermal noise density $(=-174 \mathrm{dBm} / \mathrm{Hz})$ and noise figure $(\mathrm{dB})$, respectively, and $B W$ is receiver noise bandwidth $(\mathrm{Hz})$ "'[2].

For LTE, it is therefore as indicated in Eqn. (6).

$$
N=-174 \frac{\mathrm{dB} \mathrm{m}}{\mathrm{Hz}}+N_{\text {figure }}+10 \log (12 \times 15000 \mathrm{~Hz} \times R B)
$$

As presented in [2] " $\mathrm{RB}$ is number of victim receiver physical resource blocks and is equal to $6,25,50,75$ and 100 for receiver bandwidths $(1.4,5,10,15$ and 20) $\mathrm{MHz}$ respectively" [2]. According to 3GPP [10], LTE can tolerate maximum $1 \mathrm{db}$ degradation in receiver sensitivity.

The $A C L R$ and $A C S$ values using methodology from [7], [10], [12].

\section{ORTHOGONAL FREQUENCY DIVISION MULTIPLEXING (OFDM)}

Orthogonal Frequency Division Multiplexing (OFDM) is a modulation scheme that extends the concept of single subcarrier modulation by using multiple subcarriers within the same single channel. In this case, the signal spectrum related to different subcarriers will overlap in the frequency domain but the time domain wave forms of the subcarriers are orthogonal. Four causes of degradation in performance of wireless communication networks are mainly delay spread, noise, interference, and channel variation. OFDM technique allows available bandwidth to be used efficiently and omitting any inter-carrier interference.

OFDM systems offers high data because it mixes up multiple low date rates with subcarriers with long symbol duration. Severe inter-symbol interference (ISI) arises when transmit symbol interval is not as long as the delay spread.

OFDM prevents inter symbol interference (ISI) usually arises when signals of symbol are short duration on a multipath channel MIMO-OFDM communication system. Contrary to single carrier systems, OFDM counteracts the high mobility outdoor channels by transmitting simple constellation at low symbol rates. As a result, the OFDM signal is resistant to noise, interference and delay spread that is a major concern in operation of wireless communications networks.

For the implementation of OFDM systems the use of Discrete Fourier Transform (DFT) decreased to perform baseband modulation and demodulation which was eliminated the banks of coherent demodulators and sub carrier oscillators required by frequency division multiplexing. DFT based frequency division multiplexing can be fully implemented in digital baseband. FFT for highly efficient processing, a fast algorithm for computing DFT, can even further reduce the number of arithmetic operations to $N \log N$ from $N 2$ ( $N$ if FFT size).

A guard interval can be used in between consecutive symbols and the raised cosine windowing in the time domain to reduce or eliminate the ISI and the ICI. Nevertheless, over a time dispersive channel the system could not maintain perfect orthogonality between subcarriers. This problem was tackled with the use of cyclic prefix (CP) or cyclic extension. Here they are replaced in the guard interval with a cyclic extension of the OFDM symbol. The ISI can be eliminated completely if the length of cyclic extension is longer than impulse response of the channel. Further, this scheme simulates well with a channel performing cyclic convolution which ensures the orthogonality between subcarriers over a time dispersive channel. Main idea of OFDM system is division of high data rate stream into a number of low data rate streams which is all transmitted over narrow sub channels at the same time. Therefore, it has both a frequency modulation and frequency division multiplexing.

In OFDM the main cause of signal degradation is other cell 
interference and background noise due to smart way of one tap equalization by the addition of cyclic prefix. The signal to noise plus interference is given by following formula (7),

$$
\rho_{O F D M}=\frac{P}{F P+N o}
$$

where $P$ is power, $F$ represents the ratio of other cells and own cell signal. Therefor the Shannon Capacity formula $C=B \log _{2}(1+S N R)$ becomes,

$$
\begin{gathered}
C=\log _{2}\left(1+\frac{P}{F P+N o}\right) \\
C_{O F D M}=\log _{2}\left(1+\frac{\rho}{\rho F+1}\right)
\end{gathered}
$$

where,

$$
\rho=\frac{P}{N o} \text { is signal to Noise plus interference ratio. }
$$

In OFDM, we need to take cyclic prefix factor into an account. So OFDM capacity Eqn.(9) is scaled down by the factor, as given below

$$
C_{O F D M}=\left(1-\frac{\Delta}{T_{S}}\right) \log _{2}\left(1+\frac{\rho}{\rho F+1}\right)
$$

where $\Delta$ is a cyclic prefix duration and $T_{s}$ is the OFDM symbol duration.

\section{MIMO SYSTEMS}

Multiple Input and Multiple Output (MIMO) systems is usage of multiple antennas including both transmitter and receiver to improve wireless communication performance (see Fig. 2). It is mostly used for both uplink and downlink scenarios [14].
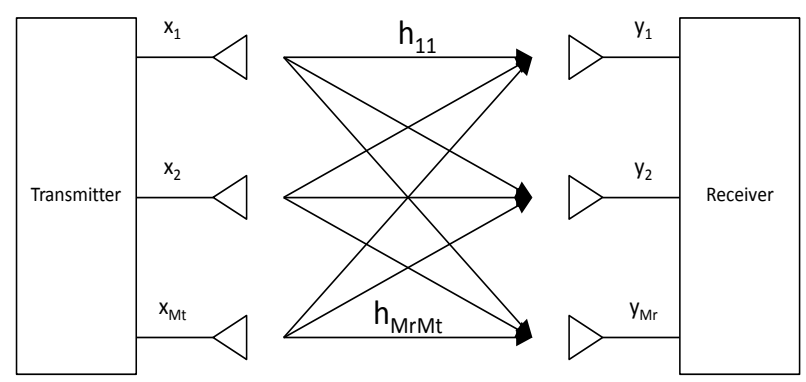

Fig. 2. MIMO systems.

For MIMO system, the capacity is given by Shannon formula (10),

$$
C=\log _{2} \operatorname{det}\left(I+\frac{1}{\sigma_{n}^{2}} H R_{x} H^{H}\right)
$$

where $H$ represents complex channel gain which incorporates fading influence of channel, $R_{x}$ is the convariance matrix of input signal $x(t)$ and $\sigma_{n}^{2}$ is the variance of uncorrelated Gaussian noise. The Shannon Capacity is the fundamental limit for error free communication. As by the property of determinant [15],

$$
\operatorname{det}(I+A B)=\operatorname{det}(I+B A)
$$

The Eqn.(13) becomes,

$$
C=\log _{2} \operatorname{det}\left(I+\frac{1}{\sigma_{n}^{2}} R_{x} H H^{H}\right)
$$

where $H H^{H}$ is a positive definite matrix [16], with eigen values $\sigma_{1}, \sigma_{2}, \ldots, \sigma_{r}$. It is diagonalized using the unitary matrix such as $H H^{H}=W \sum W^{H}$ where $W$ is unitary matrix whose columns are associated eigenvectors, such that the Eqn.(12) becomes,

$$
C=\log _{2}\left\{\operatorname{det}\left[I+\frac{1}{\sigma_{n}^{2}} R_{x} W \sum W^{H}\right]\right\}
$$

By applying the property of determinant Eqn.(13),

$$
C=\log _{2}\left\{\operatorname{det}\left[I+\frac{1}{\sigma_{n}^{2}} \sum W^{H} R_{x} W\right]\right\}
$$

The capacity of equivalent parallel uncoupled MIMO systems has been proved by [17] as follows:

$$
C=\sum_{k=1}^{N} \log _{2}\left\{\left(1+\frac{1}{\sigma_{n}^{2}} \sigma_{k}^{2} P_{k}\right)\right\}
$$

\section{MIMO OFDM SYSTEM}

Several researches have shown potential in extending OFDM systems to much higher data rates by adopting MIMO smart antenna technique system [1]. Fig. 3 shows the MIMO-OFDM system with $N$ subcarriers used over MIMO frequency selective channel [17]. The fading coefficients are spatially uncorrelated over frequency and they remain constant in time. Input data is in frequency domain, it is digitally modulated using any modulation scheme. The data is fed into Alamouti time space encoder and code words are created following the Alamouti coding scheme [18].

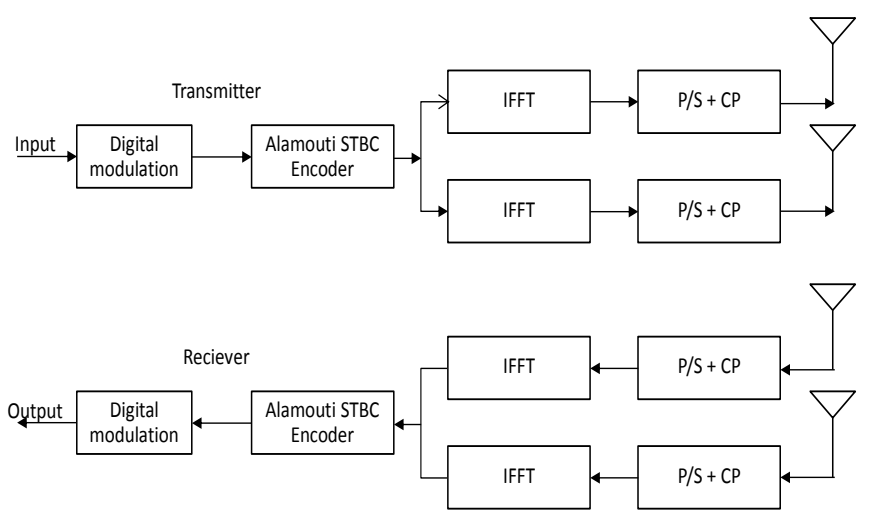

Fig. 3. MIMO OFDM block diagram.

"The data is converted in time domain by taking IFFT of code words generated by Alamouti encoder. Then the transmitter appends the cyclic prefix" [18]. After adding cyclic prefixes the Alamouti coded symbol are transmitted by the two antennas in the first instant. In second instant, other Alamouti coded symbol are transmitted. At the receiver cyclic prefix are removed from OFDM Alamouti code symbols, then they are converted in frequency domain by 
taking FFT. The output of FFT block is fed into Alamouti docorder which decouples the desired symbol form unwanted symbols according to Alamouti decoding [18]. Finally the data is digitally demodulated to detect the correct symbol which was sent initially to get the output symbol.

\section{Simulation AND RESUlts}

In this section, we evaluate the performance of Alamouti's $2 \times 2$ MIMO-OFDM systems on coexistence of FDD-LTE base stations. The simulation results are demonstrated between Signal to Noise Ratio (SNR) and Symbol Error Rate (SER) for 4QAM modulation in the presence of Rayleigh fading. Simulation parameters chosen are listed in Table II. The $5 \mathrm{MHz}$ interferer bandwidth is maintained constant throughout simulation. In this research, we separate two cells of 1 antenna element at $10 \mathrm{~km}$.

TABLE II: SIMULATION PARAMETERS

\begin{tabular}{ll}
\hline \hline Parameter & Value \\
\hline Frequency & $2.6 \mathrm{GHz}$ \\
Constellation & $4 \mathrm{QAM}$ \\
FFT size & 64 \\
Cyclic Prefix size & $15 \%$ of FFT size \\
Delay Spread & $\mathrm{CP}$ size -1 \\
Channel Model & Rayleigh fading single tap \\
Noise Model & Rayleigh noise \\
Distance between antennas & $10 \mathrm{~km}$ \\
\hline \hline
\end{tabular}

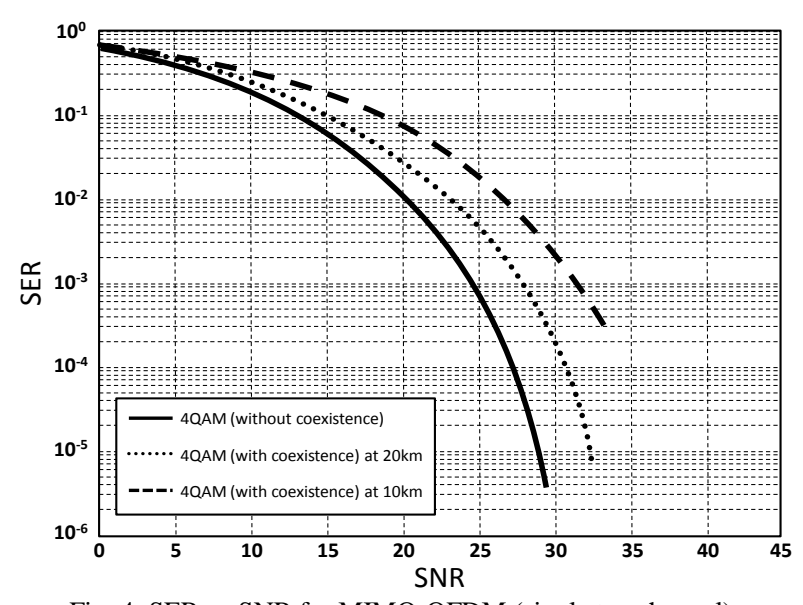

Fig. 4. SER vs SNR for MIMO-OFDM (single tap channel).

Fig. 4 shows the SER values as a function of varying SNR for the MIMO-OFDM system with Rayleigh fading single tap by using 4QAM modulation. Simulation results show that the performance of MIMO-OFDM system in this study is degraded by the coexistence interference.

\section{CONCLUSION}

At the same time that high data rates and high mobility in wireless network is in high demand, engineers currently face challenges in omitting delay, spread, noise, interference and channel variation in operation of wireless communications. This research adopted OFDM and MIMO to eliminate inter-carrier interference in aiming that any available bandwidth will be used efficiently. In order to increase the potential in reaching higher data rates, MIMO smart antenna is also adopted. Nevertheless, the results of this research indicate that the performance of MIMO-OFDM system in this study is degraded by the coexistence interference.

\section{REFERENCES}

[1] K. W. Suh and J. S. Jang, "A study on compatibility analysis based on minimum coupling loss applicable to frequency coordination combined with geographic information," International Journal of Multimedia \& Ubiquitous Engineering, vol. 8, 2013.

[2] A. Oudah and N. Seman, "On the impact of MIMO antennas on collocation and coexistence requirements of LTE networks in $2.6 \mathrm{GHz}$ frequency band," International Journal of Multimedia \& Ubiquitous Engineering, vol. 8, 2013.

[3] ECCREPORT119, "Co-existence between mobile systems in the 2.6 $\mathrm{GHz}$ frequency band at the FDD/TDD boundary," Electronic Communications Committee, 2008

[4] H. Biao, T. Haifeng, W. Wen, F. Jian, and Z. Na, "Coexistence studies for LTE-FDD with TD-LTE in the band 2500-2690 MHz," in Proc. IET International Conference on Communication Technology and Application, 2011, pp. 411-416.

[5] C. Xiang, J. Xiaowei, P. Moorut, R. Love, S. Yakun, and X. Weimin et $a l$. , "Coexistence analysis involving 3GPP long term evolution," in Proc. 2007 IEEE 66th Vehicular Technology Conference, 2007, pp. 225-229.

[6] ITU-R, "M.2243: assessment of the global mobile broadband deployments and forecasts for international mobile telecommunications," 2011.

[7] I. Parker and S. Munday. Assessment of LTE $800 \mathrm{MHz}$ base station interference into DTT receivers. ERA Technical Report 2011-0351. [Online]. Available: http://stakeholders.ofcom.org.uk/ binaries/consultations/dtt/annexes/Ite-800-mhz. pdf2011.

[8] P. Chang et al., "Interference analysis and performance evaluation for LTE TDD system," in Proc. 2010 2nd International Conference Advanced Computer Control, 2010, vol. 5, pp. 410-414.

[9] H. Sari, G. Karam, and I. Jeanclaude, "Transmission techniques for digital terrestrial TV broadcasting," IEEE Commun, Mag., vol. 33, no. 2, pp. 100-109, Feb. 1995.

[10] 3GPP TS 25.942, "Evolved Universal Terrestrial Radio Access Network (E-UTRAN); Radio Frequency (RF) system scenarios (Release 8),” 2008.

[11] ITU-R P.452-12, "Prediction procedure for the evaluation of microwave interference between stations on the surface of the earth at frequencies above about $0.7 \mathrm{GHz}, " 2005$.

[12] 3GPP, "Evolved universal terrestrial radio access (E-UTRA); Base station (BS) radio transmission and reception (release 8)," 2008.

[13] K. Farooq, LTE for 4G Mobile Broadband, Air Interface Techologies and Performance, Cambridge University Press, UK, pp. 30, 2010.

[14] D. Gredory, Durgin, Space-Time Wireless Channels, Pearson Prentice Hall, USA, p. 234, 2003.

[15] C. E. Shannon, "A mathematical theory of communication," The Bell System Technical Journal, vol. 27, issue 3, pp. 379-423, July 1948.

[16] G. Strange, Introduction to Linear Algebra, Wellesley Cambridge Press, USA, pp. 321-330, 1993.

[17] H. Majeed, R. Umar, and A. A. Basit, "Smart antennas - MIMO, OFDM \& single carrier FDMA for LTE," Degree project, Linnaeus University, 2011.

[18] S. M. Alamouti, "A simple transmit diversity technique for wireless communications," IEEE J. Sel. Areas Comm., vol. 16, pp. 1451-1458, Oct. 1998.

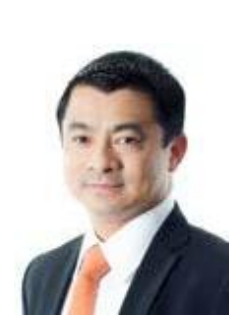

Settapong Malisuwan was born on March 24, 1966 in Bangkok, Thailand. He received his $\mathrm{PhD}$ degree in electrical engineering (telecommunications), specializing in EMI/EMC from Florida Atlantic University (State University System of Florida), Boca Raton in 2000. He received an MSc degree in electrical engineering in mobile communications system, from George Washington University in 1996, an MSc in electrical engineering from Georgia Institute of Technology in 1992 and a BSc in electrical engineering from the Chulachomklao Royal Military Academy, Nakhon-Nayok, Thailand in 1990. He served in the Royal Thai Armed Forces for more than 25 years. His research interests are in efficient spectrum management and telecommunications policy and management. Col. Dr. Settapong Malisuwan is currently the elected vice chairman and board member in the National Broadcasting and Telecommunications Commission, Thailand. 


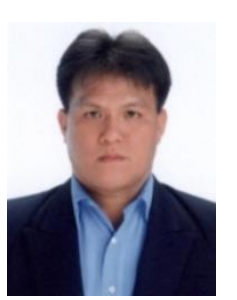

Jesada Sivaraks was born on May 12, 1970 in Bangkok, Thailand. He received his Ms.EE degree from Oklahoma State University in 1996 and BEng from King Mongkut"s Institute of Technology, Thailand. He completed his $\mathrm{PhD}$ in electrical engineering at Florida Atlantic University, Boca Raton, FL in 2001. Since 2011, he has been working in National Broadcasting and Telecommunications Commission as the secretary to the vice chairman. His $\mathrm{PhD}$ work is on the system aspects of Bluetooth, WLAN and Mobile IP/CDPD. His current research interests are in telecommunication planning and related system analysis and efficient spectrum management. He is a member of Tau Beta Pi, Florida Epsilon and was an honorary advisory's chairman of Science \& Technology committee of Parliament in 2009.

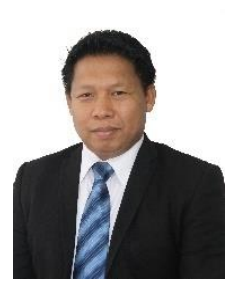

Noppadol Tiamnara was born on November 12, 1968 in Pah Na Korn Sri Ayuttaya, Thailand. He received the degree diploma of Vocational Education in Industrial Technology from South-East Asia Colledge, Thailand, 1990, and the bachelor degree of electrical engineering from Saint John's University, Thailand, 2002. He received the master degree of master of science (technology management) from Thammasart University, Thailand, 2012. Since 2006, he has been working in National Broadcasting and Telecommunications Commission as assistant of vice chairman of National Broadcasting and Telecommunication Commission (NBTC). His research interests include LTE design, wireless systems, microstrip antenna and applied electromagnetic.

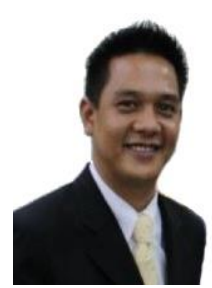

Yodsapon Thamachareon was born in Bangkok, Thailand on Feb. 13, 1976. He received his bachelor of accountant from Dhonburi Rajabhat University 1998. He has been working as an assistant to vice chairman in National Broadcasting and Telecommunications, Bangkok, Thailand since November 2012. His research interests are in technology management and spectrum management. 